\title{
Fully Ion-Implanted InP JFET with Buried p-Layer
}

SUNG J. KIM, MEMBER, IEEe, JICHAI JEONG, MEMBER, IEEE, G. P. VELLA-COLEIRO, MEMBER, IEeE, AND P. R. SMITH

Abstract-A buried p-layer has been successfully implemented in a fully ion implanted InP JFET for the first time. Using Be co-implanted with $\mathrm{Si}$, a sharp channel profile is obtained. The saturation current has been reduced and the pinch-off characteristic has been improved with a slight decrease in transconductance and cutoff frequency. The equivalent circuits for the JFET with and without the buried p-layer are compared.

\section{INTRODUCTION}

TN GaAs MESFET fabrication, buried $p$-layers have been Lintroduced to improve the threshold-voltage uniformity [1], [2], to alleviate the short-channel effects [3], to suppress the drain conductance transients [4], and to enhance the immunity to radiation [5].

The fabrication of fully implanted InP JFET's [6] requires a higher energy channel implant than that of GaAs MESFET's, because the channel exists under the $\mathrm{p}^{+}$-gate rather than under the gate metal. The higher energy implant results in larger channeling tails which are further broadened by diffusion during the high-temperature activation cycle.

While higher channel doping can be effective in enhancing device characteristics [7], it also raises the saturation current and makes the pinch-off voltage of the depletion-mode device more negative. These changes are undesirable for low-power analog or digital switching applications.

In this paper, we report on the first application of the buried p-layer to the fabrication of a fully implanted InP JFET to obtain devices with low saturation current and small pinch-off voltage without affecting other aspects of them.

\section{Buried P-Implant}

First the channel doping profile was evaluated. A deep Be implant was used to form the buried p-layer under a Si channel implant. Fig. 1 shows the doping profiles obtained from a Polaron electrochemical profiler. When the $220-\mathrm{keV} \mathrm{Si}$ implant was used alone with a dose of $1 \times 10^{13} \mathrm{~cm}^{-2}$, the profile had a broadened tail as in curve $(a)$. When a $150-\mathrm{keV}$ Be was co-implanted, the profile became abrupt. For a dose of $5 \times 10^{12} \mathrm{~cm}^{-2}$ (shown by curve $(b)$ ), a p-doped layer was detected under the $\mathrm{n}$-channel. For a reduced dose of $1 \times 10^{12}$ $\mathrm{cm}^{-2}$ (shown by curve $(c)$ ), however, the p-layer was absent through compensation and carrier depletion by the built-in junction potential.

In fabricating the JFET, a procedure identical to that

Manuscript received August 17, 1989; revised November 8, 1989.

S. J. Kim was with AT\&T Bell Laboratories, Murray Hill, NJ 07974. He is now with the Department of Electronics Engineering, Seoul National University, Seoul, Korea.

J. Jeong, G. P. Vella-Coleiro, and P. R. Smith are with AT\&T Bell Laboratories, Murray Hill, NJ 07974.

IEEE Log Number 8933325.

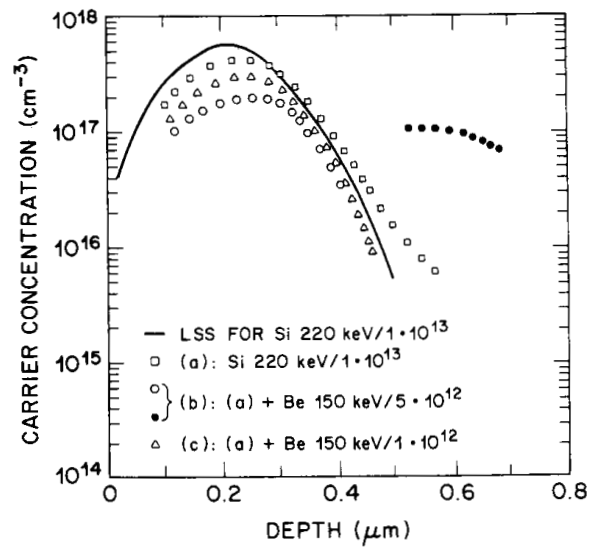

Fig. 1. Channel doping profiles measured using Polaron profiler: $(a)$ when Si $220 \mathrm{keV} / 1 \times 10^{13} \mathrm{~cm}^{-2}$ implant was used alone; $(b)$ when Be $150 \mathrm{keV} /$ $5 \times 10^{12} \mathrm{~cm}^{-2}$ was co-implanted; and (c) when Be $150 \mathrm{keV} / 1 \times 10^{12} \mathrm{~cm}^{-2}$ was co-implanted. The filled circles represent the p-type buried doping found in $(b)$. Also shown is the LSS profile for $(a)$.

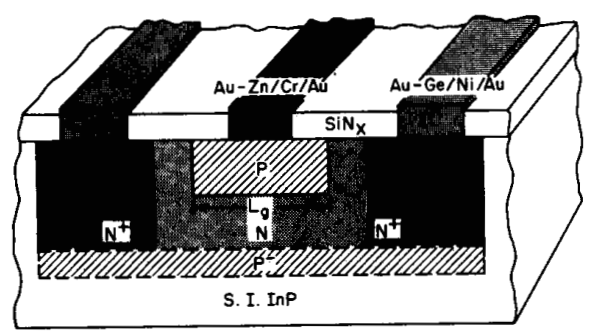

Fig. 2. Cross-sectional view of the InP JFET fabricated with buried p-layer.

described in [6] has been used except that the buried $\mathrm{Be}$ implant was added during the channel implant step. Fig. 2 shows a schematic cross-sectional view of the buried p-layer InP JFET. The p-layer is dotted to show that all the charges there are depleted.

\section{Device Results}

Fig. 3(a) and (b) shows the typical current-voltage characteristics of the FET's fabricated without and with the buried player, respectively. The FET's had a gate of $1.5 \mu \mathrm{m}$ length and $100 \mu \mathrm{m}$ width. The standard (without buried p-layer) FET had a saturation current of $150 \mathrm{~mA} / \mathrm{mm}$ and a pinch-off voltage of $-3.2 \mathrm{~V}$, while those of the buried p FET were $65 \mathrm{~mA} / \mathrm{mm}$ and $-1.7 \mathrm{~V}$, respectively. The transconductance $g_{m}$, however, has been degraded slightly from 90 to $70 \mathrm{mS} / \mathrm{mm}$. This may be due to the slight decrease in doping concentration (Fig. 1). Our Hall measurement showed little change in the channel electron mobility with the buried p-implant. The degradation 


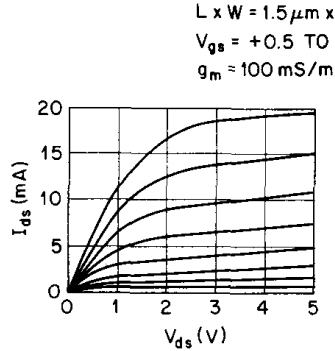

(a)
$-\mathrm{xW}=1.5 \mu \mathrm{m} \times 100 \mu \mathrm{m}$

$V_{0 \mathrm{~s}}=+0.5$ TO $-3 \mathrm{~V}$ WITH O.5V STEP

$g_{m}=100 \mathrm{mS} / \mathrm{mm}$ PER DIV.

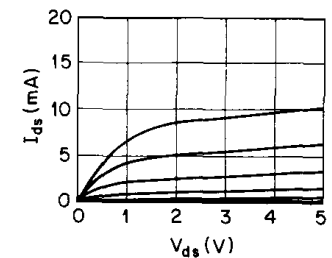

(b)
Fig. 3. FET $I-V$ characteristics for JFET's (a) without and (b) with buried p-layer. The FET had a gate width of $100 \mu \mathrm{m}$.

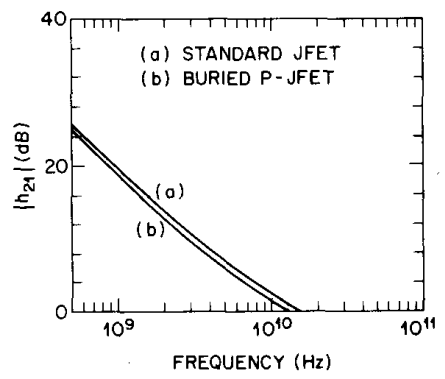

Fig. 4. Short-circuit current gain $\left(h_{21}\right)$ of the JFET's $(a)$ without and $(b)$ with buried p-layer. The unity gain frequency is $10 \mathrm{GHz}$ for $(a)$, and $8 \mathrm{GHz}$ for $(b)$.

of $g_{m}$ is responsible for the small reduction in the unity gain cutoff frequency $f_{T}$ from 10 to $8 \mathrm{GHz}$ as can be seen in Fig. 4 . The short-circuit current gain $\left(h_{21}\right)$ was calculated from microwave $S$-parameter measurements using a wafer prober from Cascade Microtech connected to a HP 8510 network analyzer.

From the same set of data, the parameters for the equivalent circuit of the JFET in Fig. 5 were derived using an optimization program [8] and tabulated in Table I. Except for $g_{m}$, the two equivalent circuits are very similar to each other, with the difference within the measurement error. The gate resistance $R_{g}$ in both devices is relatively large because of high p-contact resistance in the gate. The p-ohmic metal and p-layer itself add extra gate resistance, but these are much smaller than contact resistance. It should also be noted that the parasitic capacitances are basically unchanged, which confirms that the buried p-layer is totally depleted.

\section{CONCLUSIONS}

A fully implanted InP JFET has been fabricated for the first time with a buried p-layer. The saturation current and the

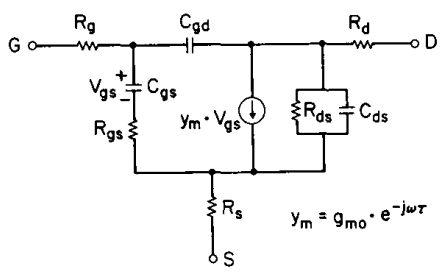

Fig. 5. Equivalent circuit for the JFET's.

TABLE I

EQUIVALENT CIRCUIT (IN FIG 5) PARAMETERS FOR THE STANDARD JFET AND THE JFET WITH BURIED P-LAYER. THE WIDTHS OF THE JFET's WERE $100 \mu \mathrm{m}$.

\begin{tabular}{|c|c|c|c|c|c|c|c|c|c|c|}
\hline & $\begin{array}{l}\mathrm{C}_{\boldsymbol{g}} \\
{[\mathrm{fF}]}\end{array}$ & $\begin{array}{l}\mathrm{C}_{\mathrm{gd}} \\
\text { [fF] }\end{array}$ & $\begin{array}{l}C_{d s} \\
\text { [fF] }\end{array}$ & $\begin{array}{l}\mathbf{R}_{\mathbf{E}} \\
{[\mathbf{\Omega}]}\end{array}$ & $\begin{array}{c}\mathbf{R}_{\mathrm{d}} \\
{[\mathbf{\Omega}]}\end{array}$ & $\begin{array}{c}\mathbf{R}_{\mathbf{t}} \\
{[\Omega]}\end{array}$ & $\begin{array}{l}\mathbf{R}_{\mathbf{g}} \\
{[\boldsymbol{\Omega}]}\end{array}$ & $\begin{array}{l}\mathbf{R}_{\mathrm{ds}} \\
{[\mathbf{\Omega}]}\end{array}$ & $\underset{[\mathbf{m S}]}{\mathbf{g}_{\mathbf{m}}}$ & $\underset{[\mathrm{ps}]}{\tau}$ \\
\hline Standard & 106 & 23 & 30 & 137 & 15 & 22 & 11 & 651 & 9.4 & 6.7 \\
\hline Buried p & 90 & 28 & 20 & 146 & 20 & 29 & 13 & 752 & 7.3 & 8.1 \\
\hline
\end{tabular}

pinch-off voltage have been drastically reduced, beneficial for low-power applications. The transconductance and the cutoff frequency have been slightly degraded with the equivalent circuit basically unchanged otherwise.

\section{ACKNOWLEGMENT}

The authors would like to thank G. Guth, J. Eng, and D. DeBlis for help in processing test samples.

\section{REFERENCES}

[1] Y. Umemoto, S. Takahashi, N. Matsunga, M. Nakamura, and K. Ueyanangi, "GaAs MESFETs with a buried p-layer for large scale integration," in Proc. 1982 GaAs IC Symp., p. 173.

[2] R. Anholt and T. Sigmon, "Substrate-impurities effects on GaAs MESFETs," J. Electron. Mater., vol. 17, no. 1, p. 5, 1988.

[3] K. Yamasaki, N. Kato, and M. Hirayama, "Below 10ps/gate operation with buried p-layer SAINT FETs," Electron. Lett., vol. 20, no. 25/ 26, p. 1029 , Dec. 1984.

[4] P. Canfield and L. Forbes, "Suppression of drain conductance transients, drain current oscillations, and low-frequency generationrecombination noise in GaAs FETs using buried channels," IEEE Trans. Electron Devices, vol. ED-33, no. 7, p. 925, July 1986.

[5] Y. Umemoto, N. Masuda, J. Shigeta, and K. Mitsusada, "Improvement of alpha-particle-induced soft-error immunity in a GaAs SRAM by a buried p-layer,"'IEEE Trans. Electron Devices, vol. 35, no. 3, p. 268, Mar. 1988.

[6] S. Kim et al, "A low-power high-speed ion-implanted JFET for InPbased monolithic optoelectronic IC's," IEEE Electron Device Lett. vol. EDL-8, p. 518, Nov. 1987.

[7] S. Kim, G. Guth, and G. Vella-Coleiro, "Integrated amplifiers using fully ion implanted InP JFET's with high transconductance," IEEE Electron Device Lett., vol. 9, no. 6, p. 306, June 1988.

[8] S. E. Sussman-Fort, CiAO Manual, SPEFCO Software, Stony Brook, NY, 1987. 